\title{
Birth preparedness and complication readiness among women in Mpwapwa district, Tanzania
}

\author{
DAVID P. URASSA ${ }^{1 *}$, ANDREA B. PEMBE ${ }^{2}$ and FATUMA MGANGA ${ }^{3}$ \\ ${ }^{1}$ Department of Community Health, Muhimbili University of Health and Allied Sciences, P.O. Box 65015, Dar \\ es Salaam, Tanzania \\ 2Department of Obstetrics and Gynaecology, Muhimbili University of Health and Allied Sciences, Dar es \\ Salaam, Tanzania \\ ${ }^{3}$ Expanded Programme on Immunisation, Ministry of Health and Social Welfare, Dar es Salaam, Tanzania
}

\begin{abstract}
Avoidable mortality and morbidity remains a formidable challenge in many developing countries like Tanzania. Birth preparedness and complication readiness by mothers are critical in reducing morbidities and mortalities due to these complications. The aim of this study was to assess knowledge and practices with respect to birth preparedness and complication readiness among women in Mpwapwa district in Tanzania. A total of 600 women who became pregnant and or gave birth two years preceding the survey were interviewed. Among them $587(97.8 \%)$ attended antenatal clinic (ANC) at least once during their last pregnancy. Two thirds of those who attended ANC made four or more visits. The median gestation age at booking for antenatal care was 16 weeks. However, $73.9 \%$ the women booked after 16 weeks of gestation. Two thirds of the women were 20-34years old and had at least primary education level. Three hundred and forty six $(57.7 \%)$ had parity between two and four. Only $14.8 \%$ of the women knew three or more obstetric danger signs. The obstetric danger signs most commonly known included vaginal bleeding during pregnancy $(19 \%)$, foul smelling vaginal discharge (15\%) and baby stops moving (14.3\%). The majority $(86.2 \%)$ of the women had decisions made on place of delivery, a person to make final decision, a person to assist during delivery, someone to take care of the family and a person to escort her to health facility. Majority (68.1\%) of the women planned to be delivered by skilled attendant. One third of the women planned to deliver at home in the absence of a skilled birth attendant. In the bivariate analysis, age of the woman, education, marital status, number of ANC visits and knowing $\geq 3$ obstetric danger signs were associated with birth preparedness and complication readiness. In multivariate logistic regression analysis, women with primary education and above were twice more likely to be prepared and ready for birth and complications. Women who knew $\geq 3$ obstetric danger signs were 3 times more likely to be prepared for birth and complications. In conclusion, women with higher level of education and those who knew obstetric complications were more prepared for birth and complications. Further studies are recommended to find out why women do not prepare for birth or complications especially that need blood transfusion.
\end{abstract}

Key words: Birth preparedness, Complication readiness, rural district, Tanzania

\section{Introduction}

Every pregnant woman is at risk of pregnancy complications which are unpredictable and can lead to morbidity or mortality of herself or her baby (JHPIEGO, 2004). Hence, the concept of birth preparedness and complication readiness in which the family and community should have an advanced planning and preparation to ensure safety and well being of the women and newborns throughout pregnancy, delivery and after delivery. Good plans and preparations will increase utilization of skilled care and reduce delays in accessing care in case of pregnancy and delivery complications.

\footnotetext{
* Correspondence: David P. Urassa: E-mail: durassa2@yahoo.co.uk
} 
Birth preparedness and complication readiness strategy encourage women to be informed of danger signs of obstetric complications and emergencies, choose a preferred birth place and attendant at birth, make advance arrangement with the attendant at birth, arrange for transport to skilled care site in case of emergence, saving or arranging alternative funds for costs of skilled and emergency care, finding a companion to be with the woman at birth or to accompany her to emergency care source. Other measures include identifying a compatible blood donor in case of haemorrhage, obtaining permission from the head of household to seek skilled care in the event that a birth emergency occurs in his absence and arrange a source of household support to provide temporary family care during her absence (JHPIEGO, 2004). The Tanzanian government has increased efforts on ensuring implementation of the birth preparedness and complication readiness by introducing focused antenatal care (FANC) which emphasizes counselling of women on the elements of this strategy (MoHSW, 2002). Tanzania has a maternal mortality rate of 454 per 100, 000 live births (TDHS, 2010). Several strategies have been initiated nationally to address burden of maternal morbidity and mortality. However little is known about factors affecting mothers' status of birth preparedness and complication readiness in Mpwapwa and in Tanzania as a whole. Mpwapwa is one of the districts with relatively high maternal and infant mortality rate (Kwesigabo et al., 2008), despite the district health management team emphasis on provision of FANC. This study was designed to assess the coverage of birth preparedness and complication readiness and its associated factors among women in Mpwapwa district, Tanzania.

\section{Material and Methods}

\section{Study area}

Mpwapwa is one of the six districts of Dodoma capital city of Tanzania. The district covers a total area of 7,479 $\mathrm{km}$ and has a population of about 269,850 projected from the 2002 population and houses census (NBS, 2002) with estimated annual growth rate of $2.1 \%$. The population is predominantly rural. The health institutions in the district include one district hospital, two health centres and 40 dispensaries. All heath institutions provide antenatal and delivery care services.

\section{Study design and data collection}

A sample size of 576 women was computed by Epi info 6 computer programme with the assumption that, since there was no previous data for birth preparedness of nearby districts, the percentage of women practicing birth preparedness and complication readiness could be $50 \%$, the confidence interval was $95 \%$, a margin of error of 0.05 and a design effect of 1.5 due to cluster sampling of wards.

Through a multistage cluster random sampling a total of 600 women from the whole district were selected to be included in the study. Administratively the district was divided into 3 divisions, 18 wards and 84 villages. Aiming at getting representative sample for the district, wards in each division were sorted according to their population and the top two with higher number of population from each division were picked for the study and treated as clusters. All women in the community who reported to be pregnant in the past two years at the time of data collection, in selected wards of Mpwapwa district were included regardless of the outcome of their pregnancies. For those who had more than one pregnancy within the last 2 years only the last pregnancy was considered. 
In June 2005, the pre-trained research assistants with midwifery skills collected data using a modified interview schedule recommended by JHPIEGO to collect information on birth preparedness and complication readiness (JHPIEGO, 2004). The interview schedule included information on age of the respondent, marital status, education level, occupation, income and number of deliveries. Other information collected was on elements of birth preparedness and complication readiness and antenatal care attendance in the last pregnancy. The antenatal cards were reviewed where available to confirm the obstetric information and the gestational age the woman booked for antenatal care.

The selected wards were visited for one day before data collection to explain about the purpose of the study and request permission to conduct the study from the ward leaders. On the day of data collection the ward and village leaders assisted to identify the households. The research assistants interviewed eligible women using the Swahili translated interview schedule after getting oral informed consent. No woman refused to participate.

\section{Data analysis}

Data was entered and analysed by Statistical Package for Social Sciences (SPSS) computer programme. The results were summarized into frequency tables. A woman was considered to be prepared for birth and its complication if she reported that she or her family had identified place of delivery, saved money and identified a means of transport to the health facility in case of obstetric emergencies ahead of childbirth (2). Bivariate analysis was done to identify factors associated with birth preparedness and complication readiness and those found significant were entered in the multivariate logistic regression analysis. The results are presented as odds ratio (OR) and 95\% confidence interval (CI).

\section{Ethical considerations}

Ethical clearance was requested and obtained from The Senate Research and Publication Committee of Muhimbili University of Health and Allied Sciences also permission to conduct the study was requested and granted from Mpwapwa district Administrative Officer. Informed verbal consent was requested and obtained from each individual woman after informing them about the study. All of them agreed to participate.

\section{Results}

A total of 600 women were enrolled into the study. Among them 587 (97.8\%) attended antenatal clinic at least once during their last pregnancy. Two thirds of those who attended antenatal care made four or more visits. Five hundred and three $(95.9 \%)$ of the women had antenatal cards at their homes used during the last pregnancy at the time of interview. The median gestation age at booking for antenatal care was 16 weeks (Range: 8-32), however 434 $(73.9 \%)$ women booked after 16 weeks of gestation.

Out of 600 women $89(14.8 \%)$ knew three or more obstetric danger signs. The obstetric danger signs more commonly known to women were vaginal bleeding during pregnancy $(19 \%)$, foul smelling vaginal discharge $(15 \%)$ and baby stops moving $(14.3 \%)$. Fewer women knew that larger abdomen than normal, labour longer than 12 hours, cord prolapse and convulsion as obstetric danger signs (Table 1). 
Table 1: Knowledge of danger signs in pregnancy and labour $(\mathrm{N}=600)$

\begin{tabular}{lll}
\hline Danger sign in pregnancy and labour & Number & \% \\
\hline Fatigue & 12 & 2 \\
Breathlessness & 10 & 1.7 \\
Baby stop moving & 86 & 14.3 \\
Foul smelling vaginal discharge & 90 & 15 \\
Vaginal bleeding during pregnancy & 114 & 19 \\
Heavy bleeding after delivery & 45 & 7.5 \\
Large abdomen than normal & 4 & 0.7 \\
Convulsions & 8 & 1.3 \\
Labour longer than12 hours & 6 & 1 \\
Headache & 21 & 3.5 \\
Swelling of face, hands, legs and feet & 18 & 3 \\
Cord prolapse & 6 & 1 \\
High fever & 83 & 13.8 \\
Pallor & 64 & 10.7 \\
\hline
\end{tabular}

Multiple responses

The majority $(86.2 \%)$ of the women had decisions made on place of delivery, a person to make final decision, a person to assist during delivery, someone to take care of the family and a person to escort her to health facility as well as other birth preparedness and complication readiness (Table 2$)$. However, only 52 (8.7\%) women arranged for a potential blood donor.

Table 2: Practices of women on birth preparedness and complication readiness $(\mathrm{N}=600)$

\begin{tabular}{|c|c|c|}
\hline Birth plan & Number & $\%$ \\
\hline Decision on place of delivery & 583 & 97.2 \\
\hline Choose a person to assist during delivery & 517 & 86.2 \\
\hline Arrange skilled attendance at delivery & 436 & 86.2 \\
\hline Arrangement for a potential blood donor & 52 & 8.7 \\
\hline Savings in case of emergency & 536 & 89.3 \\
\hline $\begin{array}{l}\text { Identify the decision making process in case of } \\
\text { obstetric emergency }\end{array}$ & 564 & 94.0 \\
\hline Arrange temporary family care during emergency & 548 & 91.3 \\
\hline $\begin{array}{l}\text { Identify and arrange transportation in case of } \\
\text { emergency }\end{array}$ & 494 & 82.3 \\
\hline Identify a person to escort during emergency & 559 & 93.2 \\
\hline Prepare birth supplies for delivery & 535 & 89.2 \\
\hline
\end{tabular}

Table 3: Decision on assistant at delivery and place of delivery

\begin{tabular}{llll}
\hline Birth plan & Response & Number & Percent \\
\hline Attendant at delivery $^{* *}$ & Skilled attendant & 377 & 68.1 \\
& TBA & 161 & 29.1 \\
& Other & 16 & 2.9 \\
Place of delivery $^{* *}$ & Home & 186 & 33.6 \\
& Dispensary & 191 & 34.5 \\
& Health centre & 18 & 3.2 \\
& Hospital & 145 & 26.2 \\
\hline
\end{tabular}

\footnotetext{
** 46 women did not have delivery plans
} 
Majority $(68.1 \%)$ of the women planned to be delivered by skilled attendant. One out of three women planned to deliver at home in the absence of a skilled birth attendant rather than in the health facilities (Table 3).

Two thirds of the women were between the age of 20 and 34 and had at least primary education level. A majority were peasants and were either married or cohabiting. Out of 600 three hundred and forty six (57.7\%) had parity between two and four (Table 4$)$. In the bivariate analysis the independent variables age of the woman, education, marital status, number of antenatal care visits and knowing $\geq 3$ obstetric danger signs were associated with birth preparedness and complication readiness. When all these independent variables were included in multivariate logistic regression analysis, women with primary education and above were twice more likely to be prepared and ready for birth and complications. Furthermore women who knew $\geq 3$ obstetric danger signs were 3 times more likely to be prepared for birth and complications (Table 4).

Table 4: Bivariate' and multivariate logistic regression analysis of the likelihood of the woman to be prepared for birth and complications

\begin{tabular}{|c|c|c|c|c|c|}
\hline Variable & Response & Total & Preparedness & $\begin{array}{l}\text { Bivariate } \\
\text { OR }(95 \% \mathrm{CI})\end{array}$ & $\begin{array}{l}\text { Multivariate } \\
\text { OR }(95 \% \mathrm{CI})\end{array}$ \\
\hline \multirow[t]{3}{*}{ Age (years) } & $<20$ & 116 & 91(78.4) & $1.4(0.7-2.8)$ & $\begin{array}{l}1.6(0.7-3.5) \\
\end{array}$ \\
\hline & $20-34$ & 410 & $350(85.4)$ & $2.3(1.3-4.1)$ & $1.7(0.9-3.4)$ \\
\hline & $\geq 35$ & 74 & $53(71.6)$ & 1 & 1 \\
\hline \multirow[t]{2}{*}{ Education } & No education & 213 & $161(75.6)$ & 1 & 1 \\
\hline & $\begin{array}{l}\text { Primary education } \\
\text { and above }\end{array}$ & 387 & $333(86.0)$ & $2.0(1.3-3.04)$ & $2.1(1.3-3.3)$ \\
\hline \multirow[t]{2}{*}{ Occupation } & Peasant & 508 & $418(82.3)$ & 1 & \\
\hline & Other & 92 & $76(82.6)$ & $1.02(0.57-1.84)$ & \\
\hline \multirow[t]{3}{*}{ Marital status } & Single & 83 & $64(77.1)$ & $1.6(0.62-4.1)$ & $1.7(0.6-4.5)$ \\
\hline & Married/cohabiting & 489 & $411(84.0)$ & $2.5(1.1-5.7)$ & $3.0(1.3-7.2)$ \\
\hline & Divorced/Widowed & 28 & $19(67.9)$ & 1 & 1 \\
\hline \multirow[t]{3}{*}{ Parity* } & 1 & 117 & $98(83.2)$ & $1.4(0.8-2.7)$ & \\
\hline & $2-4$ & 346 & $291(84.1)$ & $1.5(0.9-2.4)$ & \\
\hline & $\geq 5$ & 134 & $134(78.4)$ & 1 & \\
\hline No. of ANC & $<4$ & 211 & $168(79.6)$ & 1 & 1 \\
\hline visits $^{* *}$ & $\geq 4$ & 376 & $323(85.9)$ & $1.6(1.0-2.4)$ & $1.5(0.9-2.3)$ \\
\hline Daily income & $<1000$ & 400 & $324(81.0)$ & 1 & \\
\hline (TShs) & $\geq 1000$ & 200 & $170(85.0)$ & $1.3(0.84-2.1)$ & \\
\hline Obstetric & $<3$ & 511 & $410(80.2)$ & 1 & 1 \\
\hline danger signs & $\geq 3$ & 89 & $84(94.4)$ & $4.1(1.6-10.5)$ & $3.2(1.2-8.2)$ \\
\hline
\end{tabular}

*Three women had no parity indicated; ${ }^{* *} 13$ women did not attend antenatal care

\section{Discussion}

In this study majority of the women (97.8\%) had attended antenatal care at least once and among those attended two-thirds made four or more visits. Almost $74 \%$ of the women booked for antenatal care after 16 weeks of pregnancy. Few women were aware of three or more obstetric danger signs. One out of three women who had decisions on place of delivery planned to deliver at home in the absence of the skilled attendant. Women with primary education and those who knew three or more obstetric danger signs were more likely to be 
prepared for birth and complications compared to women with no education and those who knew less than three obstetric danger signs.

Early booking and regular attendance to antenatal care in the course of pregnancy are important in monitoring the physical status of the women and the foetus, detecting diseases and complications and providing appropriate treatment and care. Furthermore, it gives an opportunity for the woman to be counselled and make an appropriate plan for delivery (WHO, 1994). Despite of the majority of the women attended antenatal care at least once and about two-thirds at least four times but a substantial proportional of them booked for antenatal care after the 16 weeks of pregnancy. These findings are similar to the one reported in the Tanzania Demographic and Health Survey of 2010/5 (TDHS, 2010) and other developing countries (Ebeigbe \& Igberase, 2005; van Eijk et al., 2006; Hiluf \& Fantahun, 2008). The late booking for antenatal care may hinder provision of some preventive and treatment measures during pregnancy.

Majority of the women had decisions made on critical components of birth preparedness and complication readiness including a person to assist during delivery, someone to take care of the family, person to escort her to the hospital and place of delivery. One-third of women who had decisions on a place of delivery intended to delivery at home. Moreover, only $14.8 \%$ of the women were aware of three or more obstetric danger signs which signify a low awareness. Low awareness may be a cause of failure to recognize the complication when it occur thus delaying the decisions to seek care (Thaddeus \& Maine, 1994; JHPIEGO, 2004). Decision to deliver at home and low awareness of obstetric danger signs are serious bottleneck in the reduction of maternal mortality as home delivery may be associated with more delay to get appropriate care when a complication occurs (Gabrysch \& Campbell, 2009). There is a need to investigate further why women prefer to delivery at home instead of using the health facilities.

Women with primary education and above were twice as much likely to be prepared for birth and complications compared to those with no formal education. Furthermore women who knew three or more obstetric danger signs were three times more likely to be prepared for birth and complications. The findings correlate well with the other study done in Ethiopia where the literate women were about 2 times more prepared for birth than the illiterate ones (Hiluf \& Fantahun, 2008). This might be related to the fact that educated women are more likely to have better income and negotiating power in their societies thus making their own decision in matters related to their health. Limitations of these findings could be due to the fact that information related to the level of birth preparedness and complication readinesses were obtained through interviews. However, what people say may not necessarily be what they practice and this may limit generalisation of these findings

In conclusion, women with more education and those aware of obstetric complications were more prepared for birth and complications if emerged than illiterate women. Education about preparation for birth and its complication and empowerment of women are important factors in enhancing birth preparedness and hence reducing the effect of pregnancy related complications though these were not studied. Antenatal care clinics which are well attended should give due emphasis to preparation for birth and its complication and provide information and education to all pregnant women. Further studies are warranted to find out why women were prepared less for obstetric danger signs that would need blood transfusion. 


\section{Acknowledgements}

We would like to thank Mr. R. Ngadaya, Mrs. F. Msambili, Mr. S. Sembuche and Mr. J. Phillipo for data collection. This study received financial support from the World Health Organization Tanzania Country Office and the Ministry of Health and Social Welfare, Tanzania.

\section{References}

Ebeigbe, P.N. \& Igberase, G.O. (2005) Antenatal care: a comparison of demographic and obstetric characteristics of early and late attenders in the Niger Delta, Nigeria. Medical Science Monitor 11, CR529-32.

Gabrysch, S. \& Campbell, O.M. (2009) Still too far to walk: literature review of the determinants of delivery service use. BMC Pregnancy Childbirth 9, 34.

Hiluf, M. \& Fantahun, M. (2008) Birth preparedness and complication readiness among women in Adigrat town, north Ethiopia. Ethiopian Journal Health Development 22,14-20.

JHPIEGO (2004) Monitoring birth preparedness and complication readiness: tools and indicators for maternal and newborn health. Baltimore, JHPIEGO.

Kwesigabo, G., Urassa, D.P. \& Mpembeni. R. (2008) Comprehensive Study to assess Capacity of the Health System to Deliver Maternal and Newborn Services in Dodoma Region. UNICEF Dar es Salaam, Tanzania.

MoHSW (2002) Focused, antenatal care, malaria and syphilis in pregnancy: Orientation package for service providers. Dar es Salaam, Ministry of Health and Social welfare, The United Republic of Tanzania.

NBS (2002) Population and Housing Census, Dar es Salaam, Tanzania, National Bureau of Statistics (NBS) and ORC Macro.

TDHS (2010) Tanzania Demographic and Health Survey 2010, Dar es Salaam, Tanzania, National Bureau of Statistics (NBS) and ORC Macro.

Thaddeus, S. \& Maine, D. (1994) Too far to walk: maternal mortality in context. Social Science \& Medicine 38, 1091-110.

van Eijk, A.M., Bles, H.M., Odhiambo, F., Ayisi, J.G., Blokland, I.E., Rosen, D.H., Adazu, K., Slutsker, L. \& Lindblade, K.A. (2006) Use of antenatal services and delivery care among women in rural western Kenya: a community based survey. Reproductive Health 3, 2.

WHO (1994) Mother-Baby Package: Implementing Safe Motherhood in Countries. Practical Guide. Geneva, World Health Organization. 